\title{
AN INTUITIONISTIC DEFINITION OF CLASSICAL NATURAL NUMBERS
}

\author{
VLADIMIR LIFSCHITZ
}

\begin{abstract}
A definition of natural numbers in the theory of species is given which allows us to prove intuitionistically all theorems of classical arithmetic. This provides an alternative to the well-known Gödel negative translation.
\end{abstract}

In this note we give a definition of natural numbers in the theory of species [1] which allows us to prove intuitionistically all theorems of classical arithmetic. This definition leads to a translation of classicial arithmetic into the theory of species, which, unlike well-known Gödel-type translations, does not change the logical form of translated formulae.

Recall that a species $X$ is stable if $\forall x(\neg \neg X x \rightarrow X x)$; this formula is abbreviated as $\operatorname{St}(X)$. We define $\mathrm{CN}(X)$ (" $X$ is a classical (natural) number") by $\operatorname{St}(X) \& \neg \neg \exists x(X=\{x\})$. The theorem below shows that the species satisfying this condition have essentially the same properties as natural numbers in classical arithmetic.

If natural numbers are identified with singleton species, then the property $\mathrm{CN}$ is weaker than being a natural number. Thus the collection of classicial natural numbers can be looked upon as an extension of the collection of natural numbers of constructive mathematics. It has often been said that the meaning of the existential quantifier in constructive mathematics is different from its classical meaning. The above remarks suggest that this difference is perhaps more "quantitative" than "qualitative": classical quantifiers simply restrict variables to a certain extension of the constructive system of natural numbers.

These remarks also suggest that both classical and constructive theories of natural numbers can be embedded into a more general system of arithmetic. Such a system is sketched in the abstract [2] and will be discussed in detail elsewhere.

HAS is the theory of species, $A_{2}$ is classical second-order arithmetic. For every primitive recursive function symbol $f$, let $f^{*}$ be the function symbol in HAS defined by

$$
f^{*} X_{1} \cdots X_{k}=\lambda y \forall x_{1} \in X_{1} \cdots \forall x_{k} \in X_{k}\left(f x_{1} \cdots x_{k}=y\right) .
$$

Received by the editors January 19, 1979.

AMS (MOS) subject classifications (1970). Primary 02C15, 02E05.

Key words and phrases. Intuitionistic logic, theory of species. 
For every first-order term $r$, let $r^{*}$ be the second-order term obtained from $r$ by substituting distinct species variables for all its parameters and replacing every function symbol $f$ by $f^{*}$. For every first-order formula $F$, let $F^{*}$ be the formula of HAS obtained from $F$ by substituting distinct species variables for all free and bound variables, replacing each function symbol $f$ by $f^{*}$, and restricting each quantifier to $\mathrm{CN}$.

The double negation translation $F^{\prime}$ of a first-order formula $F$ is the result of inserting $\neg \neg$ before every $\exists$ in $F$. (We assume that disjunction is eliminated in favor of $\exists$, as in [1, 1.3.7].)

THEOREM. For every first-order sentence $F$,

(i) $\vdash_{\text {HAS }} F^{*} \leftrightarrow F^{\prime}$,

(ii) $\vdash_{\mathrm{A}_{2}} F$ iff $\vdash_{\mathrm{HAS}} F^{*}$.

The proof is based on the following lemmas, where $\vdash$ stands for $\vdash_{\text {HAS }}$.

LEMMA 1. For every first-order term $r\left(x_{1}, \ldots, x_{n}\right)$, all parameters explicitly shown:

(i) $\vdash \&_{i=1}^{n} \mathrm{CN}\left(X_{i}\right) \rightarrow \mathrm{CN}\left(r^{*}\left(X_{1}, \ldots, X_{n}\right)\right)$,

(ii) $\vdash r^{*}\left(\left\{x_{1}\right\}, \ldots,\left\{x_{n}\right\}\right)=\left\{r\left(x_{1}, \ldots, x_{n}\right)\right\}$.

Proof. By induction on $r$, using the derivability of $f^{*}\left\{x_{1}\right\} \cdots\left\{x_{k}\right\}=$ $\left\{f x_{1} \cdots x_{k}\right\}$.

LEMMA 2. For every formula $F(X)$ of $H A S$, if

$$
\vdash \forall X_{\mathrm{CN}(X)}(F(X) \leftrightarrow \neg \neg F(X))
$$

then

$$
\vdash \exists X_{\mathrm{CN}(X)} F(X) \leftrightarrow \neg \neg \exists X_{\mathrm{CN}(X)} F(X) .
$$

Proof. Denote the term

$$
\lambda x\left[F(\{x\}) \& \forall y_{y<x} \neg F(\{y\})\right]
$$

by $t$. Arguing in HAS, assume

$$
F(\{x\}) \& \forall y_{y<x} \neg F(\{y\}) \text {. }
$$

Then $t=\{x\}$ and hence $F(t)$. Denote $\exists x(t=\{x\}) \& F(t)$ by $G$; thus we proved that

$$
\exists x\left[F(\{x\}) \& \forall y_{y<x} \neg F(\{y\})\right] \rightarrow G .
$$

This formula implies successively

$$
\begin{gathered}
\exists x F(\{x\}) \rightarrow \neg \neg G, \\
{[\exists x(X=\{x\}) \& F(X)] \rightarrow \neg \neg G,} \\
{[\mathrm{CN}(X) \& F(X)] \rightarrow \neg \neg G,} \\
\neg \neg \exists X_{\mathrm{CN}(X)} F(X) \rightarrow \neg \neg G .
\end{gathered}
$$

Thus, assuming $\neg \neg \exists X_{\mathrm{CN}(X)} F(X)$, we derive $\neg \neg G$, i.e.

$$
\neg \neg \exists x(t=\{x\}) \& \neg \neg F(t) .
$$


On the other hand, we have $\operatorname{St}(t)$ and, consequently, $\mathrm{CN}(t)$ and $\neg \neg F(t) \rightarrow$ $F(t)$. Hence $\mathrm{CN}(t) \& F(t)$.

LEMMA 3. For every first-order formula $F\left(x_{1}, \ldots, x_{n}\right)$, all parameters explicitly shown,

$$
\vdash\left[{\underset{i=1}{n}}_{i=1} \mathrm{CN}\left(X_{i}\right)\right] \rightarrow\left[\neg \neg F^{*}\left(X_{1}, \ldots, X_{n}\right) \leftrightarrow F^{*}\left(X_{1}, \ldots, X_{n}\right)\right] .
$$

Proof. By induction on $F$. Assume first that $F$ is $r=s$; then $F^{*}$ is $r^{*}=s^{*}$. Arguing in HAS, assume $\&_{i=1}^{n} \mathrm{CN}\left(X_{i}\right)$. By Lemma 1(i), $\mathrm{CN}\left(r^{*}\right)$ and $\mathrm{CN}\left(s^{*}\right)$; hence $\operatorname{St}\left(r^{*}\right)$ and $\operatorname{St}\left(s^{*}\right)$, and it suffices to notice that $F^{*}$ is equivalent to $\forall y\left(r^{*} y \leftrightarrow s^{*} y\right)$.

The cases when the principal sign of $F$ is a propositional connective or $\forall$ are trivial (recall that $\bigvee$ is eliminated). In the case of $\exists$, use Lemma 2.

LEMMA 4. For every first-order formula $F\left(x_{1}, \ldots, x_{n}\right)$, all parameters explicitly shown,

$$
\vdash F^{*}\left(\left\{x_{1}\right\}, \ldots,\left\{x_{n}\right\}\right) \leftrightarrow F^{\prime}\left(x_{1}, \ldots, x_{n}\right) .
$$

Proof. To simplify notation, assume $n=1$. For atomic $F$ use Lemma 1(ii). The case when the principal sign of $F(x)$ is a propositional connective is trivial.

Let $F(x)$ be $\forall y G(x, y)$. Then $F^{\prime}(x)$ is $\forall y G^{\prime}(x, y), F^{*}(X)$ is $\forall Y_{\mathrm{CN}(Y)} G^{*}(X, Y)$, and, by the hypothesis of induction, $G^{*}(\{x\},\{y\}) \leftrightarrow$ $G^{\prime}(x, y)$. The implication from $F^{*}(\{x\})$ to $F^{\prime}(x)$ is trivial. Arguing in HAS, we derive from $F^{\prime}(x)$ successively

$$
\begin{gathered}
\forall y G^{*}(\{x\},\{y\}), \\
\exists y(Y=\{y\}) \rightarrow G^{*}(\{x\}, Y), \\
\mathrm{CN}(Y) \rightarrow \neg \neg G^{*}(\{x\}, Y), \\
\neg \neg F^{*}(\{x\})
\end{gathered}
$$

and, using Lemma $3, F^{*}(\{x\})$.

Now let $F(x)$ be $\exists y G(x, y)$, so that $F^{\prime}(x)$ is $\neg \neg \exists y G^{\prime}(x, y)$, and $F^{*}(X)$ is $\exists Y_{\mathrm{CN}(Y)} G^{*}(X, Y)$. Here again the implication from $F^{*}(\{x\})$ to $F^{\prime}(x)$ is trivial. Assume $G^{\prime}(x, y)$; then, by the hypothesis of induction, $G^{*}(\{x\},\{y\})$ and consequently $F^{*}(\{x\})$. Thus we derived

$$
G^{\prime}(x, y) \rightarrow F^{*}(\{x\}) \text {. }
$$

It follows that

$$
F^{\prime}(x) \rightarrow \neg \neg F^{*}(\{x\}) .
$$

By Lemma 3, $\neg \neg$ can be dropped.

Now Part (i) of the theorem is a particular case of Lemma 4. Part (ii) follows from Part (i) and from the fact that $\vdash_{\text {HAS }} F^{\prime}$ is equivalent to $\vdash_{\mathrm{A}_{2}} F$.

The examination of the proof shows that HAS can be replaced in Part (i) by much weaker systems. Consider e.g. the following restricted form of 
comprehension:

$\mathrm{CA}^{-} \quad \forall x y((A x \& A y) \rightarrow x=y) \rightarrow \exists X \forall x(X x \leftrightarrow A x)$.

Let $\mathrm{HAS}^{-}$be obtained from HAS by replacing CA by $\mathrm{CA}^{-}$. Then $F^{*} \leftrightarrow F^{\prime}$ can be derived in $\mathrm{HAS}^{-}$.

The proof-theoretic strength of $\mathrm{HAS}^{-}$is the same as that of first-order arithmetic. It would be interesting to know whether $\mathrm{HAS}^{-}$is conservative over Heyting's arithmetic HA.

\section{BIBLIOGRAPHY}

1. A. S. Troelstra, ed., Metamathematical investigation of intuitionistic arithmetic and analysis, Lecture Notes in Math., vol. 344, Springer-Verlag, Berlin-Heidelberg-New York, 1973.

2. V. Lifschitz, A conservative extension of $\mathrm{HA}^{c}$ with the E-property, Notices Amer. Math. Soc. 25 (1978), A-362.

Departmgent of Mathematics, Brigham Young University, Provo, Utah 84602

Current address: Department of Mathematics, University of Texas, El Paso, Texas 79968 